\title{
Die strategische Autonomie Europas - eine Institution im Wandel
}

\author{
C. Hoffmann
}

Eingegangen: 20. April 2021 / Angenommen: 31. Mai 2021 / Online publiziert: 12. Juli 2021

(C) Der/die Autor(en) 2021

Zusammenfassung Das Essay skizziert die Entstehung und Bedeutung des europäischen Anspruchs auf mehr Autonomie. Auf die Parameter Hard- und Soft Power beschränkt, soll anhand von Länderbeispielen herausgearbeitet werden, ob die EU ihren neuen Anspruch bereits geltend machen konnte. Zudem betrachtet der Beitrag die transatlantischen Beziehungen und China, letzteres als ideologischer Gegenspieler der EU, da diese Beziehungen einen direkten Einfluss auf die strategische Ausrichtung der EU nehmen. Abschließend werden die materiellen und politischen Ressourcen herausgearbeitet, durch die die EU an Autonomie gewinnen könnte.

Schlüsselwörter EU · China - USA · Afrika - Strategische Autonomie ·

Multilateralismus · Soft Power · Hard Power · Entwicklungspolitik

\section{Europe's Strategic Autonomy: An Institution in Transition}

Abstract The essay outlines the emergence and significance of the European claim to greater autonomy. Focusing on the parameters of hard and soft power, country examples will be used to work out whether the EU has already been able to assert its new claim. In addition, the relationship with the USA and with China - the EU's ideological adversary - are mentioned, as these have a direct influence on the strategic orientation of the EU. Finally, material and political resources promising an EU gain in autonomy will be elaborated.

Keywords EU $\cdot$ China $\cdot$ USA $\cdot$ Africa $\cdot$ Strategic autonomy $\cdot$ Multilateralism $\cdot$ Soft power $\cdot$ Hard power $\cdot$ Development policy

Dr. C. Hoffmann, MdB ( $\bowtie)$

Fraktion der Freien Demokraten im Deutschen Bundestag, Platz der Republik 1, 11011 Berlin, Deutschland

E-Mail: Christoph.hoffmann@bundestag.de 


\section{Einleitung}

Der viel diskutierte Begriff der „strategischen Autonomie“ bedeutet im Kern, dass die Europäische Union als Institution unabhängig und entschieden ihre außenpolitischen Interessen durchsetzen kann. Die EU schaffte es bisher weder unter Anwendung von Hard Power entschlossen zu reagieren noch durch Entwicklungspolitik, in Form von Soft Power, ihre strategische Autonomie auszubauen. Die EU braucht eine mentale Umstellung, die eine klare Diplomatie und Verteidigungspolitik adressiert sowie eine nachhaltige Entwicklungspolitik, die es der EU ermöglicht, durch Soft Power Interessen wahrzunehmen.

\section{Vom Multilateralismus zur strategischen Autonomie}

Das Ziel strategische Autonomie ist keine Ad-hoc-Reaktion auf jüngste Veränderungen in der internationalen Politik, sondern ein seit 2016 formulierter außenpolitischer Ansatz des Europäischen Rates (Europäisches Parlament 2020a), der sich über Jahre konkretisiert hat. Manche präferieren den Begriff ,,europäische Souveränität“, wobei beide Termini die Möglichkeit und Notwendigkeit einer europäischen, selbstbestimmten Handlungs- und Entscheidungsfähigkeit meinen (Schwarzer 2021).

Der Begriff strategische Autonomie Europas hat ihren Ursprung in der Verteidigungs- und Sicherheitspolitik. 2016 wurde dem Europäischen Rat die globale Strategie für die Außen- und Sicherheitspolitik der Europäischen Union vorgelegt. Der Schwerpunkt liegt auf Sicherheit sowie auf dem explizit formulierten Anspruch strategischer Autonomie und stellt damit eine erhebliche Umorientierung gegenüber der Europäischen Sicherheitsstrategie aus dem Jahr 2003 dar (Europäisches Parlament 2020b). 2003 beschrieb die EU erstmals in einem Dokument sicherheitspolitische Ziele sowie Herausforderungen und Strategien. Zentrale Bedrohungen waren demnach der Terrorismus, die Verbreitung von Massenvernichtungswaffen, regionale Konflikte, Staatsversagen und organisierte Kriminalität. Um die Ziele zu erreichen, setzt die EU auf einen „effektiven Multilateralismus“ sowie die Stärkung internationaler Institutionen und Rechtsnormen (bpb 2020).

Bereits vor 2016 wird eine Weiterentwicklung des Verständnisses europäischer Souveränität vom Multilateralismus hin zu einer strategischen Autonomie erkennbar. Die damalige EU-Außenbeauftragte Catherine Ashton erklärte bereits 2013, bezugnehmend auf das gescheiterte Assoziierungsabkommen mit der Ukraine, dass die Union in der Lage sein müsse als Sicherheitsgarant in seiner Nachbarschaft entschieden zu handeln, was direkte Interventionen einschließe. Strategische Autonomie müsse sich zuerst in der Nachbarschaft der Europäischen Union materialisieren (Wagner 2014).

Sechs Jahre später forderte Josep Borrell, dass Europa endlich die Sprache der Macht lernen müsse (Borrell 2020). Bezugnehmend auf die Libyen-Krise erklärte der EU-Außenbeauftragte sogar, notfalls Soldaten der Mitgliedsländer in den Krisenstaat zu entsenden, um bei der Überwachung eines angestrebten Waffenstillstandes zu helfen. Borrell erklärte: „Wenn es einen Waffenstillstand in Libyen gibt, dann muss die EU bereit sein, bei der Umsetzung und der Überwachung dieses Waffen- 
stillstandes zu helfen - eventuell auch mit Soldaten, etwa im Rahmen einer EUMission“" (Borrell, zit. n. Die Welt 2020).

Im Laufe der Zeit wird der europäische Anspruch, Interessen durch direkte Interventionen und unter Berücksichtigung militärischer Dimensionen durchzusetzen, immer deutlicher. Die strategische Autonomie Europas ist wohl nicht zwangsläufig konträr zum bisherigen effektiven Multilateralismus, aber umfasst neue Instrumente, wie den Einsatz von Soldat*innen, um europäische Interessen durchzusetzen. Dieser neue Anspruch an Autonomie hat im Ukraine-Konflikt ihren Anstoß gefunden und wurde u.a. durch den Syrienkrieg weiter gefestigt.

\section{Die Heteronomie Europas - Die Fallbeispiele Ukraine und Syrien}

Es war für die EU eine außenpolitische Blamage, als die Ukraine das angebotene Assoziierungsabkommen 2013 bis auf weiteres nicht mehr verfolgen wollte. Stattdessen strebte die Ukraine gemeinsame Handelsgespräche mit der EU und Russland an.

Das angestrebte Assoziierungsabkommen befasste sich nicht nur mit Wirtschaftsund Handelsbeziehungen und der Schaffung einer Freihandelszone, sondern auch mit politischer Zusammenarbeit. Darin wird eine enge Kooperation in der Außenpolitik, in Justiz- und Grundrechtsfragen vereinbart (lpb o.J.). Mit dem Assoziierungsabkommen versuchte die EU die Ukraine enger an sich zu binden und somit sicherheitspolitische Bedenken gegenüber Russland besser durchzusetzen.

Nach dem gescheiterten Assoziierungsabkommen gelang es der EU 2014 doch noch ein Abkommen mit der Ukraine zu unterzeichnen (lpb o.J.). Die EU hoffte durch wirtschaftlichen Aufschwung, die Westorientierung der Ukraine zu stärken (lpb o.J.) und damit ein Gegenwicht zu Russlands Einfluss zu setzen. Eigentlich sollte im September des gleichen Jahres das gesamte Freihandelspaket des Assoziierungsabkommens in Kraft treten. Doch wegen der Proteste aus Moskau wurde dieser Teil des Abkommens aufgeschoben (Berger 2015). Allein 2014 wurden der Ukraine 3,4 Mrd. $€$ an Sonderkrediten bewilligt. Ein wirtschaftlicher Aufschwung blieb wegen innerstaatlichen Problemen wie Korruption und aufgrund Russlands Sanktionen gegen die Ukraine aus.

Auch konnte die EU nicht verhindern, dass Russland die Krim völkerrechtswidrig annektierte (Bundesregierung 2021a). Die Europäische Union setzte auf Verhandlungen und Verträge, selbst wenn diese, so wie das Minsker Abkommen, immer wieder gebrochen wurden. Waffenlieferungen oder gar eine militärische Unterstützung der Ukraine wurden kategorisch ausgeschlossen (Berger 2015), obwohl die Ukraine um diese Unterstützung bat (Die Welt 2015). Die USA hingegen unterstützten seit 2014 die Ukraine auch militärisch mit ca. 320 Mio. US-\$ pro Jahr (von Osten 2019). Seitens der EU wurden einzelne Sanktionen ausgesprochen (Europäischer Rat 2020c), an Großobjekten wie Nord Stream II hält Deutschland dennoch bis heute fest. Ein weiterer Rückschlag für die Durchsetzungskraft der EU.

Auch im Syrien-Konflikt versuchte die EU durch Diplomatie am Friedensprozess mitzuwirken. Die EU sei keine Kriegspartei und unterstützt die Vereinten Nationen bei ihren Bemühungen für eine politische Lösung, heißt es auf offizieller Seite 
der EU (Europäische Kommission 2021a). Allein durch das Veto Russlands ist jedoch mit keiner Lösung seitens des Sicherheitsrats zu rechnen. Die EU bzw. die NATO schafft es weder eine friedensstiftende Rolle einzunehmen noch bei den demographischen Verschiebungen als geschlossene Einheit zu reagieren.

Unter der Federführung Deutschlands wurde 2016 das Türkei-EU-Abkommen geschlossen, das die Notwendigkeit externer Partner zur Lösung europäischer Probleme demonstriert (Bundesregierung 2021b). Ankara verspricht in dem Abkommen, alle erforderlichen Maßnahmen zu ergreifen, um neue See- und Landrouten illegaler Migration von der Türkei in die EU zu verhindern. Deutschland sowie die EU unterstützen die Türkei seit 2016 mit Millionenbeträgen. Gegen das Abkommen verstieß die Türkei im Frühjahr 2020, als sie die Grenzen zu Griechenland zeitweise für offen erklärte. Das Abkommen war aus europäischer Sicht dennoch ein Erfolg, denn es kamen insgesamt weniger Menschen auf den griechischen Inseln an (Merkur 2021).

Auch bei der 2019 staatfindenden türkischen Militäroffensive in den Kurdengebieten im Nordosten Syriens, hielt die EU an den Abkommen fest (Riegert 2019). Die USA waren bis 2019 aktiv im Syrien-Konflikt involviert. Auf Geheiß von Donald Trump wurden die US-amerikanischen Truppen aus der syrisch-türkischen Grenzregion zurückgezogen. Ein Machtvakuum entstand, welches Erdoğan nutzte, um gegen die Kurdenmilizen vorzugehen, die bislang Partner Washingtons im Kampf gegen den Islamischen Staat waren (Schlötzer 2019).

In den letzten Jahren wurde die pazifistische Haltung der EU hinterfragt. Frankreichs Präsident Macron setzte 2017 Impulse für ein gemeinsames europäisches Militärprojekt, während die deutsche Bundeskanzlerin Angela Merkel in einer Ansprache vor dem Europäischen Parlament im November 2018 erklärte: „Wir sollten [...] an der Vision arbeiten, eines Tages auch eine echte europäische Armee zu schaffen“" (Europäisches Parlament 2019a).

Beide Länderbeispiele lassen erkennen, dass die EU über effektiven Multilateralismus sowie durch Soft und Normative Power versuchte ihre Interessen durchzusetzen (Böttger 2014), auch wenn Emmanuel Macrons und Merkels Aussagen demonstrieren, dass in Zukunft die EU eine stärkere, militärische Rolle einnehmen solle. Der Erfolg dieser Strategie, gemessen an der Ist-Situation, kann bezweifelt werden. Während die USA ihre außenpolitischen Ziele auch militärisch durchsetzen, hat die EU (in Syrien und der Ukraine) eine Beteiligung abgelehnt. Da vielmals die Interessen der zwei NATO-Partner deckungsgleich sind, konnte die EU bisher davon profitieren. Sie war aber insoweit mittelbar von Washington abhängig, dass ihre sicherheits- und außenpolitischen Interessen vertreten werden. Durch die vorsichtige Diplomatie der EU und die Ablehnung jeglicher militärischen Unterstützung wurde punktuell Stabilität in und um Europa gewahrt, eine nachhaltige europäische Lösung, im Sinne einer strategischen Autonomie, konnte jedoch nicht erreicht werden.

\section{Entwicklungspolitik als Instrument der strategischen Autonomie}

Entwicklungspolitik kann als entscheidendes Instrument eingesetzt werden, um langfristig strategische Autonomie auf Grundlage kultureller Attraktivität und oder Ideo- 
logie zu gewinnen. Durch Soft Power kann die EU nachhaltig auf die Transformation afrikanischer Länder Einfluss nehmen und mittelbar Demokratie, freie Marktwirtschaft und Menschenrechte als System unterstützen. Dieses hat wiederum langfristige Vorteile, um zentrale Weltprobleme überhaupt wirkungsvoll bearbeiten zu können. Je nach Problemkontext sind die Industrieländer auf das Zusammenspiel mit unterschiedlichen Ländergruppen angewiesen, um globale Interdependenzprobleme zu bearbeiten, die sie im Alleingang nicht mehr lösen können (Messner 2010, S. 452).

Die Entwicklungspolitik der Europäischen Union nimmt daher eine Schlüsselstellung zum Ausbau der strategischen Autonomie ein. Je durchsetzungsfähiger die EU ist, je schneller globale Probleme gelöst werden können, desto einfacher ist es für die EU ihre strategische Autonomie auszubauen und zu beanspruchen. Die Ziele der europäischen Entwicklungspolitik fußen auf der Agenda 2030 sowie auf der Verteidigung von Menschenrechten und Demokratie. Die EU agiert auf internationaler Ebene und ist der weltweit größte Geber von Entwicklungshilfe (Europäisches Parlament 2019b).

Die europäische Soft Power hat jedoch in den letzten Jahren an Wirkungskraft verloren. Innerhalb der EU konnte ein deutlicher Rechtsruck durch Populist*innen vermerkt werden, die Entwicklungspolitik einen nachgeordneten Stellenwert zuschreiben. Zudem gibt es keine gemeinsame verbindliche europäische Entwicklungspolitik. Jeder einzelne Mitgliedstaat der EU setzt seine eigene Entwicklungspolitik um. Dies führt dazu, dass die europäische Entwicklungspolitik stark von den Interessen ihrer Mitglieder geprägt ist, was es erschwert, eine europäische Lösung zu implementieren.

Das Instrument für Nachbarschaft, Entwicklungszusammenarbeit und Internationale Zusammenarbeit (Europäische Kommission 2020) beschreibt einen europäischen, langfristigen Haushaltsrahmen. Laut der EU wird dieser eine wesentliche Modernisierung der Außendimension des EU-Haushalts mit sich bringen. Er würde dazu beitragen, die Wirksamkeit und Sichtbarkeit des auswärtigen Handelns der EU zu verbessern, dessen Koordinierung mit den internen Politikbereichen zu stärken und der EU die notwendige Flexibilität für eine raschere Reaktion auf neue Krisen und Herausforderungen zu geben. Nichtsdestotrotz ist die europäische Entwicklungspolitik immer noch interdependent von der Entwicklungspolitik ihrer Mitgliedstaaten. Ergo, wenn die einzelnen Mitgliedstaaten der EU nicht im Sinne einer strategischen europäischen Autonomie handeln, so wird die EU von ihren eignen Mitgliedern ausgebremst.

\section{Das deutsche Negativbeispiel}

Das Beispiel der deutschen Entwicklungspolitik in Uganda ist ein Praxisbeispiel, wie Entwicklungspolitik konträr zu dem gesetzten Ziel einer strategischen europäischen Autonomie wirkt. Die deutsche Entwicklungszusammenarbeit konzentriert sich auf die Schwerpunkte Landwirtschaft und ländliche Entwicklung sowie erneuerbare Energien und Energieeffizienz. Außerdem fördert das Bundesentwicklungs- 
ministerium (BMZ) in Uganda eine gute Regierungsführung und die Wahrung der Menschenrechte (BMZ 2021).

Bei den Regierungsverhandlungen im November 2018 wurden Uganda durch die Bundesregierung 79,5 Mio. $€$ für die entwicklungspolitische Zusammenarbeit allein für die Jahre 2018 und 2019 neu zugesagt (BMZ 2021). Die Wirtschaft des Empfängerlandes ist jedoch weit unter seinen Möglichkeiten geblieben und der seit 34 Jahren amtierende Präsident Yoweri Museveni agiert immer mehr als Diktator (Mugabi 2018). Als Museveni bei den Wahlen 2020 Oppositionelle verfolgte, verurteilte das Europäische Parlament diese Entwicklungen und ,fordert die EU und ihre Mitgliedstaaten nachdrücklich auf, über öffentliche und diplomatische Kanäle zur Sprache zu bringen“ (Europäisches Parlament 2021a). Die deutsche Bundesregierung erklärte auf Nachfrage, dass ,,[n]achdem eventuelle Anfechtungen der Wahlen gerichtlich geklärt sind und sich die neue Regierung gebildet hat, [...] die Bundesregierung zusammen mit den anderen EU-Mitgliedstaaten Gespräche mit der ugandischen Regierung führen und auf den nötigen Dialog mit Opposition und Zivilgesellschaft drängen [wird]. Die weitere Zusammenarbeit mit Uganda wird in diesem Lichte in Abstimmung mit der Europäischen Union (EU) und im EU-Geberkreis auszugestalten sein“ (Auswärtiges Amt 2021).

Die EU fordert ihre Mitgliedstaaten auf zu handeln, während diese, darunter auch Deutschland, wiederum auf Rücksprache mit der EU verweist. Die Konsequenz ist, dass bis dato weder eine eindeutige Reaktion erfolgte noch Sanktionen ausgesprochen wurden (Rat der Europäischen Union 2021). Deutschland sowie andere europäische Länder bezahlen weiterhin Entwicklungsgelder an Uganda und damit mittelbar an den gerügten Staatspräsidenten, der nicht zuletzt dadurch seine Macht weiter ausbauen kann. Hinzu kommt, dass die Demokratie ideell an Schaden nimmt. Während die Demokratie in Uganda immer weiter ausgehöhlt wird, verliert auch die europäische Demokratie an Integrität. Es besteht das Risiko, dass die Demokratie nicht mehr als ein anzustrebendes System betrachtet wird, sondern als System der reichen Industriestaaten, die im Namen der Demokratie ihre Interessen durchsetzen.

\section{Ohne Wohlstand, keine Demokratie}

Viele Politolog*innen erkennen einen Kausalzusammenhang zwischen Wohlstand und Demokratie. Der Zusammenhang wird damit begründet, dass funktionierende Demokratien auf weit aufgefächerten Strukturen, auf Parlamente, Gerichte, Schulen, Universitäten, Infrastruktur und Landesverteidigung angewiesen sind. Diese Grundpfeiler der Demokratie können nur mit einem gedeckten Staatshaushalt bedient werden, der wiederum von Steuern und einer funktionierenden Wirtschaft abhängt (Blom 2017, S. 126-130).

Wohlstand und Demokratie sind daher eng miteinander verknüpft und erklären, warum wirtschaftliche Prosperität ein zweiter entscheidender Faktor ist, um durch Soft Power Einfluss zu gewinnen. Entwicklungsgelder werden jedoch vielmals nicht als Investitionen verstanden, sodass kein nachhaltiges Wirtschaftswachstum entstehen kann. Rechtsunsicherheiten, Korruption und Bürokratie erschweren zudem Investitionen ausländischer Unternehmen. Die wirtschaftlichen Schwierigkeiten Ugan- 
das, dessen autokratischer Wandel und die inkonsequente Entwicklungspolitik der EU-Mitgliedstaaten, in dem Fall Deutschland, dezimieren die Soft Power der EU und somit auch die Etablierung einer strategischen Autonomie in Afrika.

Es braucht eine klare Koordination der europäischen Entwicklungshilfe und klare Konsequenzen für den Bruch völkerrechtlicher Normen, die Demokratie und Menschenrechte bedrohen, um auf Grundlage kultureller Attraktivität und Ideologie Macht ausüben zu können. Solange die europäische Entwicklungspolitik durch die inkonsequente Praxis der einzelnen Mitgliedsstaaten beeinflusst wird, werden nationale (Sicherheits-)Interessen einen nachhaltigen wirtschaftlichen Aufschwung sowie jegliche demokratische Prozesse in Entwicklungsländern ausbremsen.

\section{Das Trump-Trauma und China}

In der Abwesenheit einer europäischen Wertevorstellung, fehlenden Investitionen und mangelnder Durchsetzungskraft entstand ein Vakuum, insbesondere in Subsahara-Afrika, welches u.a. von China gefüllt wird. China investiert massiv auf dem afrikanischen Kontinent in dessen Infrastruktur - Flughäfen, Straßen, Eisenbahnen, Energietrassen. Präsident Xi Jinping kündigte 2018 zum Auftakt des China-AfrikaGipfels in Peking an, 60 Mrd. US-\$ in Afrika zu investieren, wobei 15 Mrd. US\$ als „Hilfen und zinslose Kredite“ vorgesehen seien (Der Spiegel 2019). Diese Investitionen sind nicht zuletzt ein wichtiger Baustein Chinas, um das Projekt Neue Seidenstraße zu realisieren.

Im selben Jahr kündigte der damalige Kommissionspräsident Jean-Claude Juncker eine neue Afrika-Europa Allianz für nachhaltige Investitionen und Arbeitsplätze an. Das Investitionsvolumen für den Zeitraum von 2017 bis 2020 wird auf 44 Mrd. $€$ geschätzt (Europäische Kommission 2021b).

Ein weiterer Vorteil Chinas gegenüber der EU ist das Ausbleiben vergleichbarer politischer (und auch finanzieller) Beschränkungen. Dies ermöglicht agileres und vor allem schnelleres Handeln (Fox 2018). Einige Wirtschaftsexpert*innen warnen auch vor den chinesischen Krediten, da es ein erhöhtes Risiko gibt, dass die Empfängerländer sich verschulden (Diekhans 2020). Es ist ungeklärt, was die Konsequenz Pekings wären, sollten die Empfängerstaaten zahlungsunfähig sein.

Während die EU Kritik gegenüber Peking nur sehr zurückhaltend formuliert, haben die USA China nicht nur zum Konkurrenten, sondern zum Systemfeind erklärt (Rügemer 2017). Mit der Wahl Donald Trumps zum Präsidenten der Vereinigten Staaten von Amerika verschlechterte sich das chinesisch-amerikanische Verhältnis. Trump verhandelte im amerikanischen Interesse neue Freihandelsabkommen, erteilte dem Multilateralismus eine generelle Absage und trat aus mehreren Völkerrechtsverträgen sowie internationalen Organisationen aus. Seine „America-first-Politik“, die überwiegend auf amerikanische Interessen und unbedingte Überlegenheit setzt sowie sich von allgemeinen Normen und Prinzipien distanziert, begünstigt den chinesischen Führungsanspruch (Turowski 2017). Die Unterdrückung der Uiguren, die Niederschlagung der Demokratiebewegung in Hongkong und die immer offenere militärische Bedrohung Taiwans demonstrieren den Machtanspruch Chinas (Som- 
mer 2020). Das Credo „America First“ der Trump-Regierung führte paradoxerweise dazu, dass China an Macht gewinnen konnte.

Es sorgte daher für Verwunderung, als Anfang des Jahres 2021 die Verhandlungen über ein umfassendes Investitionsabkommen zwischen der EU und China im Grundsatz abgeschlossen wurden. Die Niederlande und die osteuropäischen Staaten warnten vor dem Abkommen. US-Präsident Joe Biden, der anders als sein Vorgänger Trump, eine aktive außenpolitische Rolle der USA vorsieht, formuliert deutliche Kritik an den schweren Menschenrechtsvergehen, die China in der westchinesischen Region Xinjiang verübt sowie an dem Zurückschrauben der Freiheitsrechte in Hongkong (Heide und Meiritz 2021). Auf der Münchner Sicherheitskonferenz sagte er: „Amerika ist wieder da. Das transatlantische Bündnis ist wieder da. Und wir schauen nicht zurück, wir schauen nach vorne - gemeinsam“ (Biden, zit. n. US-Botschaft 2021). Es ist ein klarer Aufruf, die transatlantischen Beziehungen zu intensivieren.

Die Wahl von Joe Biden wurde vom Europäischen Parlament begrüßt und als Chance für Europa, für die Stärkung der Beziehungen zwischen EU und USA, um gemeinsame Herausforderungen anzugehen, betrachtet. Der Präsident des Europäischen Rates, Charles Michel, sagte, ,dass die transatlantischen Beziehungen, in den letzten vier Jahren sehr gelitten haben [...]. In dieser Zeit ist die Welt komplexer, weniger stabil und weniger vorhersehbar geworden. Mehr als je zuvor erfordere dies von uns Europäern, dass wir unser Schicksal selbst in die Hand nehmen, um unsere Interessen zu verteidigen und unsere Werte zu fördern“" (zit. n. Europäisches Parlament 2021b).

Die Neuausrichtung eines transatlantischen Bündnisses sowie die Machzunahme Chinas haben für die strategische Autonomie der EU zwei Konsequenzen. Die EU muss entscheiden, ob sie mit Joe Biden die transatlantischen Beziehungen intensivieren will, oder im Sinne einer strategischen Autonomie sich weiter distanzieren. Außerdem wird die EU durch den wachsenden Einfluss Chinas gezwungen, sich zu positionieren und auf den Machtanspruchs Chinas zu reagieren.

\section{Fazit}

Die EU beansprucht, verankert in der globalen Strategie für die Außen- und Sicherheitspolitik, strategische Autonomie. Dieser Anspruch konnte aber noch nicht in der Praxis durchgesetzt werden, da die EU in entscheidenden Situationen nicht stringent handelt. Die EU hat sich durch den Verzicht auf militärische Unterstützung (Hard Power) mittelbar von den USA abhängig gemacht. Durch die Ära Trumps konnte die EU, das erste Mal seit dem Kalten Krieg, ihre Sicherheitsinteressen nicht mehr stellvertretend durch die USA wahren. Das führte zu einem immer deutlicheren Anspruch der EU, ihre Interessen auch in militärischen Dimensionen zu verteidigen.

Der Aufstieg Chinas zwingt die EU ihre strategische Autonomie zu behaupten, sodass es kaum denkbar ist, dass die EU sich gänzlich von den USA löst. Viel mehr bedarf es einer Neuausrichtung der transatlantischen Partnerschaft, in der sich die EU aktiv einbringt und Sicherheitsinteressen nicht mehr extern durchsetzen lässt.

Um in Zukunft eine strategische Autonomie zu erlangen, muss sich die EU mental umstellen und Sicherheitsfragen nachhaltig beantworten. Ohne den Einsatz von 
Hard Power, wird die EU keine komplette Autonomie erlangen und weiterhin auf amerikanische Unterstützung angewiesen sein, um ihre Interessen durchzusetzen sowie zu verteidigen.

Um über Soft Power weiter an strategischer Autonomie zu gewinnen, eignet sich insbesondere die Entwicklungspolitik. Hier bedarf es eine klare Werteverteidigung der Demokratie und nachhaltige Investitionen, die den Einwohner*innen zugutekommt. Zudem muss Soft Power als ernstes Instrument der strategischen Autonomie verstanden werden. Ergo, muss die EU die Entwicklungspolitik ihrer Mitgliedstaaten besser koordinieren. Solange die Ausrichtung der Entwicklungspolitik den Nationalstaaten unterliegt, müssen diese den Anspruch auf strategische Autonomie konsequent umsetzen.

Zusammenfassend braucht es mehr Mut, Sicherheitsinteressen durch direkte Interventionen durchzusetzen und eine konsequentere Verteidigung europäischer Werte. Nur im Zusammenspiel dieser Paradigmen wird es der EU gelingen ihren Anspruch nach strategischer Autonomie langfristig umzusetzen.

Funding Open Access funding enabled and organized by Projekt DEAL.

Open Access Dieser Artikel wird unter der Creative Commons Namensnennung 4.0 International Lizenz veröffentlicht, welche die Nutzung, Vervielfältigung, Bearbeitung, Verbreitung und Wiedergabe in jeglichem Medium und Format erlaubt, sofern Sie den/die ursprünglichen Autor(en) und die Quelle ordnungsgemäß nennen, einen Link zur Creative Commons Lizenz beifügen und angeben, ob Änderungen vorgenommen wurden.

Die in diesem Artikel enthaltenen Bilder und sonstiges Drittmaterial unterliegen ebenfalls der genannten Creative Commons Lizenz, sofern sich aus der Abbildungslegende nichts anderes ergibt. Sofern das betreffende Material nicht unter der genannten Creative Commons Lizenz steht und die betreffende Handlung nicht nach gesetzlichen Vorschriften erlaubt ist, ist für die oben aufgeführten Weiterverwendungen des Materials die Einwilligung des jeweiligen Rechteinhabers einzuholen.

Weitere Details zur Lizenz entnehmen Sie bitte der Lizenzinformation auf http://creativecommons.org/ licenses/by/4.0/deed.de.

\section{Literatur}

Auswärtiges Amt (2021). Schriftliche Fragen für den Monat Januar 2021 - Frage Nr. 1-447.

Berger, A. (2015, 29. Aug.). Zwischen Abhängigkeit und Perspektive. Deutschlandfunk. https://www. deutschlandfunk.de/die-ukraine-die-eu-und-russland-zwischen-abhaengigkeit-und.724.de.html? dram:article_id=329545. Zugegriffen: 15. März 2021.

Blom, P. (2017). Was auf dem Spiel steht. München: Carl Hanser.

BMZ - Bundesministerium für wirtschaftliche Zusammenarbeit und Entwicklung (2021). Uganda. Erneuerbare Energien, Klimaschutz und Wirtschaftspotenzial in Ostafrika. https://www.bmz.de/de/laender/ uganda. Zugegriffen: 16. März 2021.

Borrell, J. (2020, 8. Feb.). Die EU muss die Sprache der Macht neu erlernen. Der Tagesspiegel. https:// www.tagesspiegel.de/politik/europaeische-aussenpolitik-die-eu-muss-die-sprache-der-macht-neuerlernen/25524148.html. Zugegriffen: 10. März 2021.

Böttger, K. (2014). Auf dem sicherheitspolitischen Auge blind: Die EU-Außenpolitik angesichts der Ukraine-Krise: Zustand und Entwicklungsoptionen. Integration, 37(2), 95-108.

bpb - Bundeszentrale für politische Bildung (2020). Europäische Sicherheitsstrategie (ESS). https://www. bpb.de/nachschlagen/lexika/das-europalexikon/176893/europaeische-sicherheitsstrategie-ess. Zugegriffen: 11. März 2021. 
Bundesregierung (2021a). Annexion der Krim durch Russland ist und bleibt Völkerrechtsbruch. https:// www.bundesregierung.de/breg-de/aktuelles/annexion-der-krim-durch-1876950. Zugegriffen: 14. März 2021.

Bundesregierung (2021b). Fünf Jahre EU-Türkei-Erklärung. https://www.bundesregierung.de/breg-de/ suche/faq-eu-tuerkei-erklaerung-1728136. Zugegriffen: 15. März 2021.

Die Welt (2015, 18. Feb.). Ukraine fordert Waffen und härtere Sanktionen. https://www.welt.de/politik/ ausland/article137566459/Ukraine-fordert-Waffen-und-haertere-Sanktionen.html. Zugegriffen: 14. März 2021.

Die Welt (2020, 17. Jan.). Europäische Union erwägt Militäreinsatz in Libyen. https://www.welt.de/ politik/deutschland/article205118272/Buergerkrieg-Europaeische-Union-erwaegt-Militaereinsatzin-Libyen.html. Zugegriffen: 29. Febr. 2021.

Diekhans, A. (2020, 16. Juli). Wie China seinen Einfluss ausbaut. tagesschau. https://www.tagesschau.de/ wirtschaft/afrika-china-infrastruktur-coltan-101.html. Zugegriffen: 29. März 2021.

Europäische Kommission (2020). Ein EU-Haushalt für die Zukunft. https://ec.europa.eu/internationalpartnerships/system/files/factsheet-mff-multiannual-financial-framework_de.pdf. Zugegriffen: 17. März 2021.

Europäische Kommission (2021a). Europa hilft in der Syrien-Krise. https://ec.europa.eu/germany/aboutus/reasons/syria_de. Zugegriffen: 15. März 2021.

Europäische Kommission (2021b). Africa-EU Partnership. https://ec.europa.eu/info/africaeuropealliance_ de. Zugegriffen: 29. März 2021.

Europäischer Rat (2020). Restriktive Maßnahmen der EU als Reaktion auf die Krise in der Ukraine. https:// www.consilium.europa.eu/de/policies/sanctions/ukraine-crisis/. Zugegriffen: 29. März 2021.

Europäisches Parlament (2019a). Verteidigung: Kommt eine „europäische Armee“? https://www.europarl. europa.eu/news/de/headlines/security/20190612STO54310/verteidigung-kommt-eine-europaischearmee. Zugegriffen: 15. März 2021.

Europäisches Parlament (2019b). Entwicklungspolitik: ein allgemeiner Überblick. https://www.europarl. europa.eu/factsheets/de/sheet/163/entwicklungspolitik-ein-allgemeiner-uberblick. Zugegriffen: 14. März 2021.

Europäisches Parlament (2020a). Verfahren: 2019/2135(INI). https://www.europarl.europa.eu/doceo/ document/TA-9-2020-0009_DE.html. Zugegriffen: 10. März 2021.

Europäisches Parlament (2020b). Kurzdarstellungen zur Europäischen Union. https://www.europarl. europa.eu/factsheets/de/sheet/158/au\%C3\%9Fenpolitik-ziele-mechanismen-und-ergebnisse. Zugegriffen: 14. März 2021.

Europäisches Parlament (2021a). Die politische Lage in Uganda. https://www.europarl.europa.eu/doceo/ document/TA-9-2021-0057_DE.pdf. Zugegriffen: 16. März 2021.

Europäisches Parlament (2021b). Neuer US-Präsident: Hoffnung auf Neustart für transatlantische Beziehungen. https://www.europarl.europa.eu/news/de/press-room/20210114IPR95616/neuer-usprasident-hoffnung-auf-neustart-fur-transatlantische-beziehungen. Zugegriffen: 18. März 2021.

Fox, B. (2018, 5. Sep.). Investitionen in Afrika: China stellt die EU in den Schatten. Euractiv. https:// www.euractiv.de/section/eu-aussenpolitik/news/investitionen-in-afrika-china-stellt-die-eu-in-denschatten/. Zugegriffen: 15. März 2021.

Heide, D., \& Meiritz, A. (2021, 7. März). China gegen die USA: Alle Zeichen stehen auf Konfrontation. Handelsblatt. https://www.handelsblatt.com/politik/international/konflikt-der-weltmaechtechina-gegen-die-usa-alle-zeichen-stehen-auf-konfrontation/26980832.html?ticket=ST-1645380GlQcyfNXQtEgfZOYBaCV-ap2. Zugegriffen: 29. März 2021.

lpb - Landeszentrale für politische Bildung Baden-Württemberg (o. J.). Die Beziehungen der Ukraine zur EU und zur NATO. https://www.lpb-bw.de/ukraine-eu-nato. Zugegriffen: 3. März 2021.

Merkur. (2021, 16. März). Fünf Jahre Flüchtlingsdeal mit der Türkei. https://www.merkur.de/politik/fuenfjahre-fluechtlingsdeal-mit-der-tuerkei-zr-90243809.html. Zugegriffen: 14. März 2021.

Messner, D. (2010). Entwicklungspolitik. In C. Masala, F. Sauer \& A. Wilhelm (Hrsg.). Handbuch der Internationalen Politik (S. 442-460). Wiesbaden: GWV Fachverlage GmbH.

Mugabi, I. (2018). Kommentar: Yoweri Museveni - Vom Befreier zum Diktator Ugandas. Deutsche Welle. https://www.dw.com/de/kommentar-yoweri-museveni-vom-befreier-zum-diktator-ugandas/ a-45156632. Zugegriffen: 10. Juni 2021.

von Osten, D. (2019, 10. Okt.). Wer der Ukraine womit hilft. tagesschau. https:/www.tagesschau.de/ faktenfinder/ukraine-hilfsgelder-trump-faktencheck-101.html. Zugegriffen: 14. März 2021.

Rat der Europäischen Union (2021, 26. März). EU Sanction Map. https://www.sanctionsmap.eu/\#/main/ page/legend. Zugegriffen: 17. März 2021. 
Riegert, B. (2019, 19. Okt.). Die EU im Syrien-Dilemma. Deutsche Welle. https://www.dw.com/de/dieeu-im-syrien-dilemma/a-50761110. Zugegriffen: 13. März 2021.

Rügemer, W. (2017, 25. Okt.). Varianten des Kapitalismus. Ein Vergleich des westlichen mit dem chinesischen Kapitalismus. iSW - sozial-ökologische Wirschaftsforschung e.V. https://www.isw-muenchen. de/2017/10/varianten-des-kapitalismus-ein-vergleich-des-westlichen-mit-dem-chinesischen-kapita lismus/. Zugegriffen: 16. März 2021.

Schlötzer, C. (2019, 7. Okt.). USA überlassen Nordsyrien dem Spiel der Mächte. Süddeutsche Zeitung. https://www.sueddeutsche.de/politik/trump-syrien-truppenabzug-1.463074. Zugegriffen: 13. März 2021.

Schwarzer, D. (2021). Europas geopolitischer Moment. Internationale Politik, 76(1), 19-24.

Sommer, T. (2020, 22. Sept.). Nicht mit den Autoritären. Zeit Online. https://www.zeit.de/politik/2020-09/ china-usa-donald-trump-europa-eu-handel-demokratie. Zugegriffen: 10. Juni 2021.

Der Spiegel (2019, 3. Aug.). China will 60 Milliarden Dollar in Afrika investieren. https://www. spiegel.de/wirtschaft/soziales/china-verspricht-afrika-60-milliarden-dollar-hilfe-und-investitionena-1226258.html. Zugegriffen: 18. März 2021.

Turowski, J. (2017). USA vs. China: Internationale Politik als Nullsummenspiel. Zeitschrift Luxemburg. https://www.zeitschrift-luxemburg.de/usa-vs-china-internationale-politik-als-nullsummenspiel/. Zugegriffen: 10. Juni 2021.

US-Botschaft (2021, 19. Feb.). Virtuelle Münchener Sicherheitskonferenz - Rede von US-Präsident Joe Biden. https://de.usembassy.gov/de/virtuelle-muenchner-sicherheitskonferenz-2021-rede-von-uspraesident-joe-biden/. Zugegriffen: 18. März 2021.

Wagner, J. (2014). IMI - Informationsstelle Militarisierung e.V. http://edoc.vifapol.de/opus/volltexte/2014/ 5581/pdf/2014_02_wagner_web.pdf. Zugegriffen: 11. März 2021. 